\title{
Primer/Probe Optimization of RTq-PCR for Identification of Double-stranded (ds) RNA in Rhizoctonia solani
}

\author{
Mary S. Chey, Ashlee M. Long, Seema Bharathan and Narayanaswamy Bharathan \\ 114 Weyandt Hall, Biology Department, Indiana University of Pennsylvania, PA 15705, Indiana
}

\begin{abstract}
Rhizoctonia solani is a soil-borne pathogenic fungus with several distinct isolates that have been classified based on their anastomosis groups (AG's). Many isolates of these fungi contain double-stranded viral RNA (dsRNA) that are cytoplasmic and viral in origin. Research in our laboratory has studied the epidemiology and molecular biology of viral RNA in R. solani, making it a useful biological model in the development of protocols for the rapid identification of biological agents. In the present study the dsRNA from the isolate EGR-4 which is characteristically large at $3.301 \mathrm{~Kb}$ was purified. Attempts to clone middle (M)-size dsRNA fragments from $R$. solani have been very difficult primarily due to artifacts that co-purify including large (L)-size dsRNA in the fungus. Various $\mathrm{MgCl}_{2}$ concentrations were tested to optimize full length dsRNA PCR product. Magnesium is required for DNA polymerase, and EGR-4 requires a specific concentration; thus, several $\mathrm{MgCl}_{2}$ concentrations were tested. The dsRNA was analyzed by gel electrophoresis. The gel-purified, nuclease-treated dsRNA was reverse transcribed into cDNA and ligated into the p-jet cloning vector and transformed using E. coli. All such clones were sequenced and forward and reverse primers were generated using BLAST sequence via Biosearch Technology. The plasmids were purified from transformed cultures and amplified using real-time PCR (RTqPCR) with the primers (reverse CCACCGGAAGAGGGAAATCC, forward AGCGCTGACCTTGCTATCGA ATC) and probe (5' Fam-AGTGCCGATCAGCCCTCCACCG-BHQ1 3'). The ideal primer/probe concentration was determined through optimization by comparing the lowest threshold concentration $\left(\mathrm{C}_{\mathrm{t}}\right)$ values using the plasmid cDNA as a template.
\end{abstract}

Key words: Life science, Rhizoctoniasolani, double-stranded (ds) RNA, cryptic mycoviruses, phylogenetic analysis, q-PCR.

\section{Introduction}

Rhizoctoniasolani is a pathogenic fungus that has the capability of infecting a wide range of agricultural and ornamental crops. Some of the most common crops that are attacked by this fungus are rice, peanuts, strawberry, potato, tomato, grasses, cotton, wheat, tree seedlings, pine, and lettuce [1]. It primarily attacks the roots and stems. Above ground stem damage includes damping off of seedlings and "wire stem" in more mature plants. Infection of other parts of the plant can occur from the use of contaminated irrigation water. In potatoes, the fungus results in lower yield due to the pruning of young tubers. Mature tubers are damaged by sclerotia formation which presents as

Corresponding author: Seema Bharathan, Dr. Ph.D., associate professor, research field: molecular biology. E-mail: BHARATHS@iup.edu. black scurf on the surface [2]. There are many different isolates of Rhizoctoniasolani that are classified based on the anastomosis groups (AG's) [3-5]. These strains contain double-stranded RNA (dsRNA). This dsRNA is not a part of the fungus's own genome and is therefore considered to be extragenomic dsRNA. $R$. solani has hundreds of different isolates which are grouped into fourteen different anastomosis groups. Each anastomosis group is made up of $R$. solani isolates which are capable of physically touching each other, fusing and sharing materials, such as nutrients. If the isolates do not belong to the same anastomosis group, then cell death can occur at the point of physical contact of the mycelia $[4,5]$. One study, which examined 36 isolates of $R$. solani from nine anastomosis groups, found that every isolate contained double-stranded (ds) RNA. The dsRNA had a wide variation of total base pair size 
of nucleic acids and also of sequence of nucleic acids from one isolate to another. While there is some homology seen among the isolates, it was rare enough to suggest that there may be unique origins of the dsRNA for most isolates $[6,7]$. Extended observation within these studies has demonstrated from a single $R$. solani isolate that there is an array of extensive genetic diversity among dsRNA populations. Unfortunately, substantial amount of confusion lie upon the nature and origin of dsRNA in R. solani and its relationship with hypovirulence. No simple association was evident between the presences of any dsRNA species in $R$. solanias well is its degree of pathogenicity. In order to alleviate the role of dsRNA within $R$. solani, this study will be molded to developing and modifying, from several studies, protocols for dsRNA cloning. Using different types of reverse transcription enzymes, optimized salt concentrations (magnesium chloride) and TaqDNA polymerases, an attempt was made to detect dsRNA through quantitative-PCR.

\section{Materials and Methods}

\subsection{Double-Stranded RNA Extraction}

Double-stranded (ds) RNA was extracted from 15-20 $\mathrm{g}$ of $R$. solani isolate EGR4 as described previously by Bharathan and Tavantizs (1990) with few modifications, Bharathan et. al (2005). Briefly the frozen mycelium was subjected to buffer extraction and elution from CF-11 cellulose column. All such partially purified dsRNA were treated with DNase and RNase as previously described by Bharathan et. al (2005). The enzyme treated dsRNA was gel-purified and concentrated using NucleoSpin column as described by the manufacturer.

\subsection{Cloning EGR-4 dsRNA}

\subsubsection{DsRNA Ligation}

The extra pure gel-purified dsRNA was cloned using the protocol described by Potgieter et. al (2002) with few modifications. Very briefly the dsRNA was denatured with $2.5 \%(\mathrm{v} / \mathrm{v})$ DMSO and ligated with 250 ng of PC3-T7 loop primer 5'-p-GGATCCCGGG AATTCGGTAATACGACTCACTATATTTTTATA GTGAGTCGTATTA-OH-3' (Integrated DNA Technologies, USA). The ligation mixture included 50 $\mathrm{mM}$ HEPES/NaOH, pH7.9, $20 \mathrm{mM} \mathrm{MgCl}_{2}$ (Sigma), $0.01 \%$ BSA (Fermentas), 1 mM ATP (Fermentas), 3 mM DTT, 10\% DMSO, 20\% PEG $_{6000}, 20$ U Ribolock RNase Inhibitor (Fermentas), 30 U T4 RNA Ligase (Fermentas), and final volume of $30 \mu \mathrm{L}$. Ligation was accomplished in Bio-Rad Thermal Cycler at $37^{\circ} \mathrm{C}$ for $6 \mathrm{~h}, 18{ }^{\circ} \mathrm{C}$ for $1 \mathrm{~h}, 16{ }^{\circ} \mathrm{C}$ for $1 \mathrm{~h}, 14{ }^{\circ} \mathrm{C}$ for $1 \mathrm{~h}$, and 12 ${ }^{\circ} \mathrm{C}$ overnight.

\subsection{2 cDNA Synthesis}

The cDNA was reacted in a mixture containing 50 $\mathrm{mM}$ Tris/HCl, $\mathrm{pH} 8.3,3 \mathrm{mM} \mathrm{MgCl} 2,75 \mathrm{mM} \mathrm{KCl}, 10$ $\mathrm{mM}$ DTT, $1 \mathrm{mM}$ dNTPs, $20 \mathrm{U}$ Ribolock RNase inhibitor, $400 \mathrm{U}$ Maxima ${ }^{\circledR}$ Reverse Transcriptase, 5 $\mu \mathrm{L}$ of ligated sample and adding RNase-free water to the final volume of $20 \mu \mathrm{L}$. The reaction was incubated in the Biorad MyCycler Thermal Cycler at $50{ }^{\circ} \mathrm{C}$ for $30 \mathrm{~min}$ and $85^{\circ} \mathrm{C}$ for $5 \mathrm{~min}$.

\subsubsection{PCR Amplification}

For PCR amplification reaction, $5 \mu \mathrm{L}$ of cDNA was added into a mixture containing $5 \mu \mathrm{L}$ of $10 \times$ PCR buffer (AmbionRETROscript Kit: $100 \mathrm{mM}$ Tris-HCl, pH 8.3, $500 \mathrm{mM} \mathrm{KCl}, 15 \mathrm{mM} \mathrm{MgCl} 2), 2.5 \mu \mathrm{L}$ of 2.5 mMdNTP mix (AmbionRETROscript Kit), $2 \mathrm{mM}$ of $\mathrm{MgCl}_{2},-2.5 \mathrm{U}$ of SuperTaq ${ }^{\mathrm{TM}}$ Plus Polymerase (Ambion), $1.25 \mu \mathrm{M}$ of $\mathrm{PC} 2$ primer CCGAATTCCCGGGATCCand final volume of 50 $\mu \mathrm{L}$. The reaction mixture was incubated in the BioradMyCycler Thermal Cycler at $72{ }^{\circ} \mathrm{C}$ for $2 \mathrm{~min}$, $95^{\circ} \mathrm{C}$ for $2 \mathrm{~min}, 35$ cycles for $95{ }^{\circ} \mathrm{C} 25 \mathrm{~s}, 65^{\circ} \mathrm{C} 30 \mathrm{~s}, 68$ ${ }^{\circ} \mathrm{C} 2 \mathrm{~min}, 72{ }^{\circ} \mathrm{C} 10 \mathrm{~min}$.

\subsubsection{CloneJET PCR Cloning}

The PCR products were purified with GeneJETTM PCR Purification kit (Fermentas) and cloned into pDrive cloning vector (Qiagen) and transformed into $E$. coli competent cells. Next, the cloned plasmids were purified with GeneJET ${ }^{\mathrm{TM}}$ Plasmid Miniprep kit 
(Fermentas). All procedures followed the manufacturer's instructions. The purified plasmids were cut with Fermentas FastDigest ${ }^{\circledR}$ EcoRI and analyzed on a $1 \%$ agarose gel stained with ethidium bromide. After screening the correct orientation of the cloned inserts, the plasmids were sequenced using M13 forward primer and M13 reverse primer at Retrogen, Inc., USA.

\subsubsection{DNA Sequencing and Alignment}

Positive clones were picked and sent for sequencing by Retrogen, Inc.. The sequence was BLAST searched in the NCBI database. The complete sequence was formed by manual defining the overlap common sequence between the forward and reverse scanned sequence through Microsoft word "Find" program. First, the reverse complementary sequence of forward sequence was determined by using the Reverse Complement-Bioinformatics.org server (http://www.bioinformatics.org/sms/rev_comp.html).

The last row of $\sim 50$ nucleotide sequences were copied and pasted onto the "Find" program to find out the overlap sequence. It was repeated until the whole overlapping sequence had been identified. Multiple alignments of complete formed sequences were carried out using both UniProt-Sequence alignments and Clustal Omega.

\subsubsection{Design of Primer and Probe}

The final sequence was used to design the primer and probe through Primer 3 Input version 0.4.0 (http://frodo.wi.mit.edu/). The picked forward, reverse and probe primers sequences were sent to Biosearch Technologies (http://www.biosearchtech.com/) to synthesize both the forward, reverse primers and TaqManProbe. The 5' end of the TaqMan probe was labeled with fluorophores dye FAM and the 3' end with quenched dye BHQ-1.

\subsubsection{The TaqMan Probe}

The probe and primers were first tested on the cloned plasmid (where the sequences of primers and probe come from) by using SensiMix II Probe Kit from Bioline (USA). The extracted plasmid concentration was determined as in section Determination of dsRNA concentration. The optical density at $260 \mathrm{~nm}$ for DNA was $50 \mu \mathrm{g} / \mathrm{mL}$. The reaction was performed as a manufacture's protocol, but scaled to final volume of $20 \mu \mathrm{L}$. Serial dilution from $10^{-2}$ to $10^{-7} \mu \mathrm{g}$ of extracted plasmid was each mixed in reaction mixture contains 1 $\times$ of SensiMix ${ }^{\mathrm{TM}}$ II Probe, $200 \mathrm{nM}$ of forward and 600 $\mathrm{nM}$ reverse primers, $600 \mathrm{nM}$ of probe and nuclease free water up to the final volume of $50 \mu \mathrm{L}$. Blue plasmid, genomic DNA, NTC, and RT samples also ran on the same assay as a negative control. The reaction was run by Eppendorf RealPlex $^{\odot}$ Real Time PCR Thermocycler.

\section{Result and Discussion}

The double-stranded RNA of calculated size 3.3-kb was consistently detected in EGR4 contained viral dsRNA as indicated in Fig. 1, showing the characteristic high molecular weight band (3301 base-pairs).

Various concentrations of the $\mathrm{MgCl} 2$ concentrations were empirically determined to obtain optimum concentration of $\mathrm{MgCl} 2$ for cDNA preparation and cloning (Fig. 2). The optimum concentration was determined to be $2 \mathrm{mM}$. Unless otherwise mentioned in all our efforts to clone and design primers and probes 2

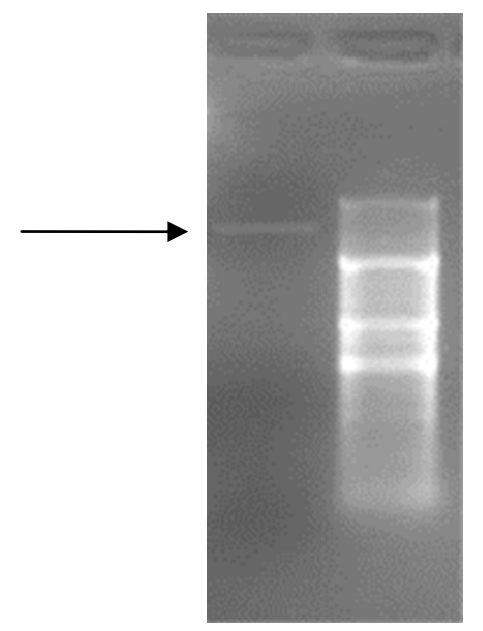

Fig. 1 Electrophoretic analysis and ethidium bromide stained nuclease treated dsRNA purified from the $R$. solani isolate EGR-4 (lane1). Lane 2 represents molecular weight markers of sizes 3,621, 2,040, 1,120, and 784 base pairs (bp). 


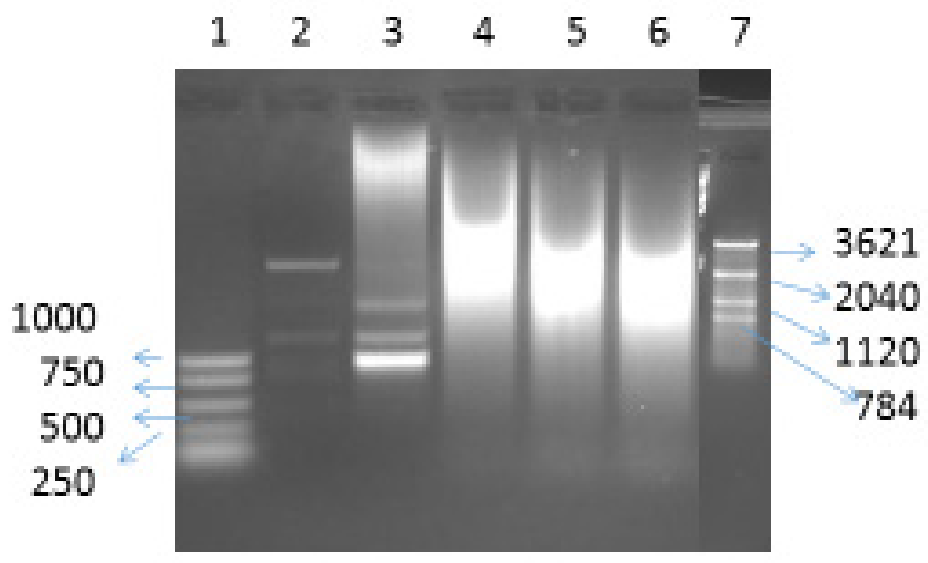

Fig. 2 Ethidium Bromide stained gel of PCR products generated from rPCR. The sizes of molecular weight markers are shown left of lane 1 molecular weight marker, lanes 2-6 represent PCR products from 2.0 and 2.5, 3.0, 3.5 and 4.0 mM concentration of $\mathrm{MgCI}_{2}$ in the PCR reaction mixture.

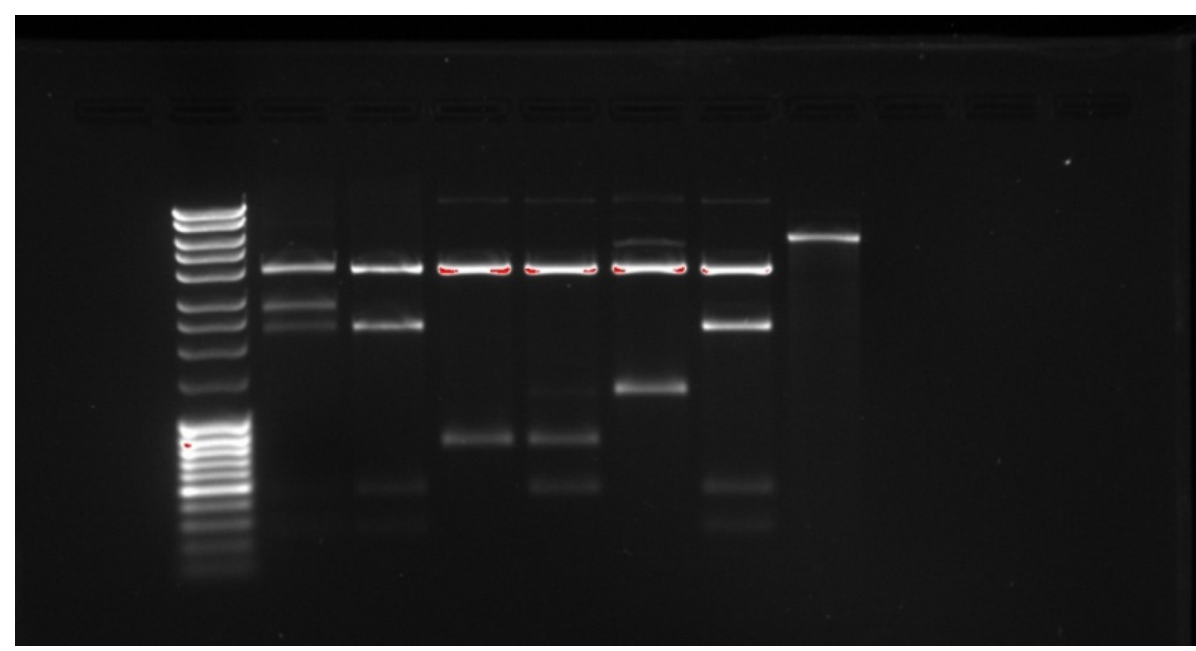

Fig. 3 Fast digest analysis of cloned PCR products onto pJet clone vector as described in materials and methods.

$\mathrm{mM}$ concentration of $\mathrm{MgCl}_{2}$ was used in all reactions. The results from our efforts to clone the PCR products are shown below Figure 3.

The isolated plasmid contained the EGR4 cDNA is shown in the Fast Digest agarose gel electrophoresis in Fig. 3. All such clones were sequenced and sequences aligned (data not shown). The obtained sequence for the

forward

(5'-AGCGCTGACCTTGCTATCGAATC-3') reverse primers (5'-CCACCGGAAGAGGGAAATCC-3') and TaqMan probe sequences were determined to be 5'-AGTGCCGATCAGCCCTCCACCG-3' with Tm values of $54{ }^{\circ} \mathrm{C}$ and $60{ }^{\circ} \mathrm{C}$, respectively. The optimized concentrations of primers were determined to $200 \mathrm{nM}$ for forward primer and $600 \mathrm{nM}$ for reverse primer. The most effective primer and probe concentrations were successfully determined for the identification of EGR4 cDNA (Fig. 4). The lowest $\mathrm{C}_{\mathrm{t}}$-FAM values were found for a primer concentration of $200 \mathrm{nM}$ and probe concentration of $150 \mathrm{nM}$, followed closely by the $400 / 150 \mathrm{nM}$ primer to probe concentration. This suggests that a lower concentration of primer and greater concentration of probe is related to greater amplification. This also suggested that the higher concentration of probe might have more of an effect than a lesser concentration of primer. The highest concentration of primer, however, at $600 \mathrm{nM}$, showed almost no amplification regardless of probe 


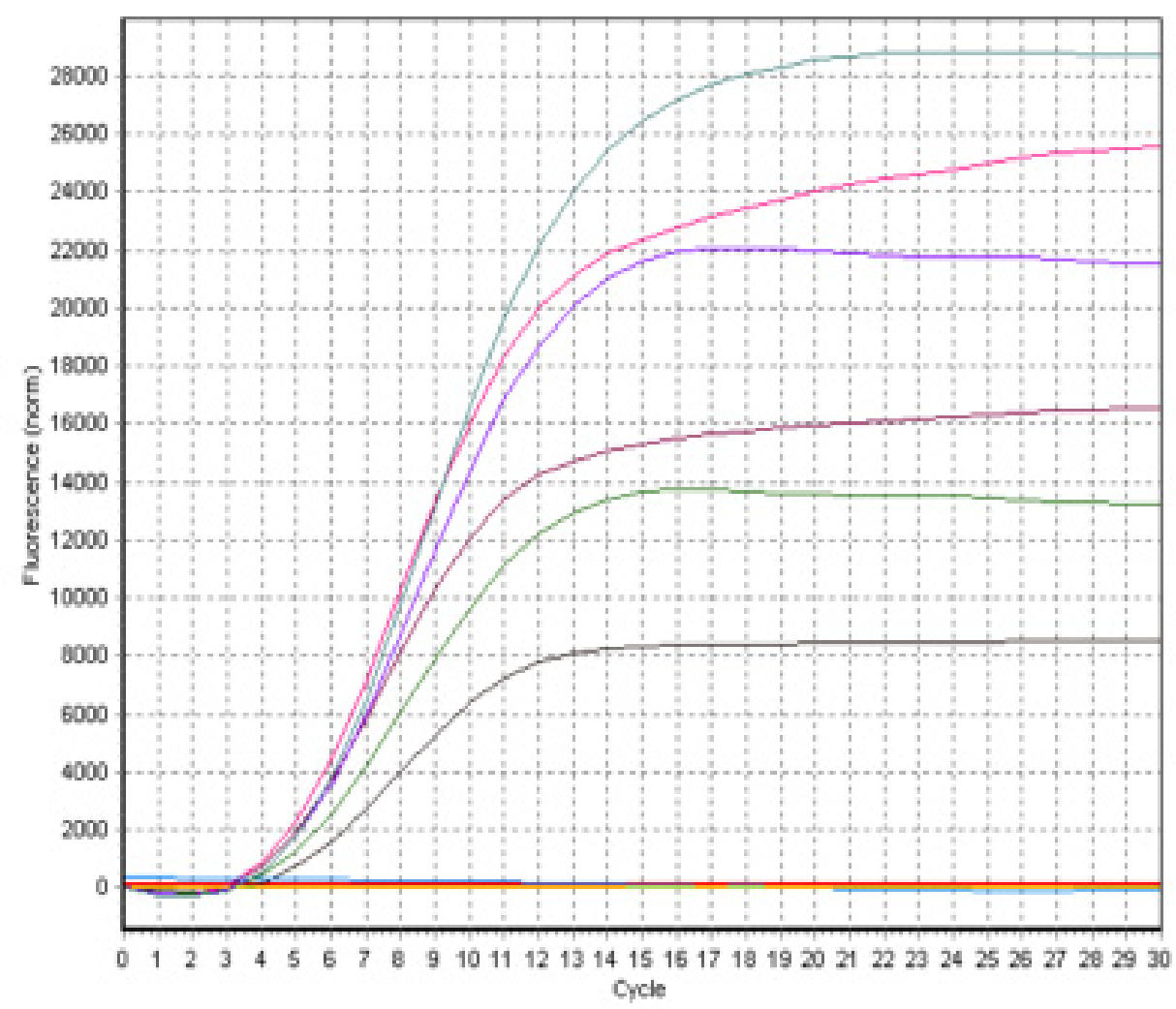

Sample/Analysis

\begin{tabular}{|c|c|}
\hline Pos & Name \\
\hline$?$ ? B2 & $200 / 50$ \\
\hline ?DB3 & $600 / 50$ \\
\hline$? \square \mathrm{C} 2$ & $200 / 100$ \\
\hline ? $\mathrm{C3}$ & $600 / 100$ \\
\hline ? D2 & $200 / 150$ \\
\hline ? D3 & $600 / 150$ \\
\hline ?】E2 & $400 / 50$ \\
\hline - E3 & NTC \\
\hline ?】F2 & $400 / 100$ \\
\hline$?$ ? G2 & $400 / 150$ \\
\hline
\end{tabular}

Fig. 4 The linear view result of primers optimization experiment. The graph showed amplification plots of all nine primer concentration combinations at $54^{\circ} \mathrm{C}$.

concentration. The NTC also showed no amplification which helped to show that there are no false positives due to any possible contamination.

Now that the signal has been optimized, the next procedures will involve comparing different $R$. solani strains to ensure specificity for the EGR4 strain and directly amplifying dsRNA using reverse transcription RT-PCR. This will permit the development of a protocol for rapidly identifying other biological agents and viruses using real-time PCR infecting other hosts. These amplification protocols were very specific and very sensitive with very little or hybridization to no template control (NTC) figure 4. We are currently testing using these forward and reverse primers and probes for mycovirus infection distribution, and detection for possible role in hypovirulence.

\section{Acknowledgments}

This research project was funded by a Senate grant from the Graduate School Research at Indiana University of Pennsylvania (IUP) to Dr. Seema Bharathan and Dr. Bharathan Narayanaswamy.

\section{References}

[1] Adams, G. C. Jr. 1988. "Thanatephorus Cucumeris (Rhizoctonia solani). A Species Complex of Wide Host Range." Advances in Plant Pathology 6: 535-52.

[2] Ogoshi, A. 1987. "Ecology and Pathogenicity of Anastomosis and Intraspecific Groups of Rhizoctonia solani Kuhn.” Annual Review of Phytopathology 25: 125-43.

[3] Bains, P., and Schaupmeyer, C. 1998. Rhizoctonia Disease of Potato (Rhizoctonia solani): Fungicidal control, susceptibility of potato cultivars and advanced breeding lines and infection of rotation crop plants with the pathogen. British Society for Plant Pathology. Edinburgh: 7th International Congress of Plant Pathology. Abstract.

[4] Carling, D. E. 1996. Grouping in Rhizoctonia solani by hyphal anastomosis. [book auth]. B Sneh, et al. Rhizoctonia Species: Taxonomy, Molecular Biology, Ecology, Pathology and Disease Control. Dordrecht: 
Kluwer Academic Publishers, pp. 37-47.

[5] Ogoshi, Akira. 1987. "Ecology and Pathogenicity of Anastomosis and Intraspecific Groups of Rhizoctonia Solani Kuhn.” Ann. Rev. Phytopathol 25: 125-43.

[6] Bharathan, N., and Tavantzis, S. M. 1990. "Genetic Diversity of Double-stranded RNA in Rhizoctonia solani." Phytopathology 80: 631-5.

[7] Bharathan, N, et. al. 2005. "Double Stranded RNA:
Distribution and Analysis among Isolates of Rhizoctonia solani AG-2 to -13." Plant Pathology (54): 196-203.

[8] Potgieter A. A., Page, N. A., Liebenberg, J., Wright, I. M., Landt, O., and Dijk, A. A. 2009. "Improved Strategies for Sequence-independent Amplification and Sequencing of Viral Double-stranded RNA Genomes." J. Gen. Virol. 90: 1423-32. 